partizipativem Journalismus: «Citizen journalism» refers to a range of web-based practices whereby <ordinary» users engage in journalistic practices» (Goode, 2009, S. 1287). Praktiken von Lai_innen, wie Blogging, das Posten von Kommentaren auf Digitalplattformen oder das Teilen von Fotos und Videos, werden in diesem Zusammenhang herausgestrichen (Goode, 2009).

Engesser und Wimmer (2009) gehen folgend davon aus, dass Gegenöffentlichkeiten (oder: kritische Teilöffentlichkeiten) aktuell auf digitale Kanäle geradezu angewiesen sind, bzw. dass diese in besonderem Masse von der Konnektivität des Internets profitieren, wonach sich Akteur_innen einfacher miteinander vernetzen können. Partizipativer Journalismus im Internet nimmt weiter eine Korrektivfunktion gegenüber dem etablierten Journalismus ein, was auch in einer klaren Gegenthematisierung erfolgen kann - dies allerdings mit vergleichsweise geringen Reichweiten (Engesser \& Wimmer, 2009). Neuberger (2012) betont in diesem Zusammenhang eine zunehmende Vermischung von Bürger_innenjournalismus, professionellem Journalismus wie auch nichtjournalistischer Kommunikation auf Digitalplattformen. Journalismus durch Lai_innen impliziert weiter eine stark normative Komponente, im Sinne einer «Verbesserung der öffentlichen Kommunikation» durch deren Partizipation, der Erfüllung deliberativer Werte für den öffentlichen Diskurs und der Möglichkeit für Bürger_innen, eine journalistische Vermittlungsrolle einzunehmen (Neuberger, 2012, S. 55). Neuberger (2012) weist jedoch gleichzeitig darauf hin, dass an den partizipativen Bürger_innenjournalismus dieselben Qualitätskriterien wie an den professionellen Journalismus herangetragen werden müssten, da dieser nur in dieser Weise ein demokratisch-funktionales Äquivalent darstellen könne.

Eine durch den medialen Wandel bedingte Ausformierung von Gegenöffentlichkeiten zeigt sich anhand des Phänomens der Alternativmedien respektive alternativen Nachrichtenmedien. Das folgende Kapitel widmet sich zentral dem Untersuchungsgegenstand der vorliegenden Arbeit.

\title{
III.4 Alternativmedien als Gegenöffentlichkeiten
}

Die in diesem Hauptkapitel geschilderten Definitionen von Gegenöffentlichkeiten und sozialen Bedingungen für deren Entstehung verdeutlichen, dass diese in unterschiedlicher Weise auftreten können. So werden häufig soziale Bewegungen oder sonstige Protestbewegungen als Beispiele für «autonome Öffentlichkeiten» aufgeführt, die sich gegen die hegemoniale Öffentlichkeit positionieren. Mit einem Blick auf die neuen sozialen Bewegungen der 1960er und 1970er Jahre zeigt sich, dass auch diese Bewegungen mit Kommunikationsmitteln wie der «Alternativpresse» gearbeitet haben (z. B. Wimmer, 2015). Aber auch schon deutlich früher, in Zeiten der Französischen Revolution, lässt sich ein Aufschwung der 
Meinungspresse im Kampf gegen die Zensur feststellen (Habermas, 2018 [1962], S. 14). Luhmann (2016 [1996], S. 75-78) äussert sich zum Begriff der «Alternative» vor allem in seinen Ausführungen zum «Protest», im Zusammenhang mit den neuen sozialen Bewegungen. Der Autor streicht dabei vor allem die Paradoxie der Begrifflichkeit heraus; so würden Protestbewegungen und die Alternativpresse beispielsweise die Gesellschaft aus einer beobachtenden Perspektive beschreiben, obgleich sie selbst Teil davon seien - weswegen Luhmann (2016 [1996], S. 76) auch formuliert: «Sie ist, wogegen sie ist.» In gleicher Weise verhält sich die Alternativpresse der neuen sozialen Bewegungen paradox, indem sie einerseits eine Kapitalismuskritik äussert, gleichzeitig aber selbst auf Finanzierung angewiesen ist. Und weiter steht die Alternativpresse als Gegenöffentlichkeit in einem ständigen Austausch mit der bürgerlichen Öffentlichkeit, gegenüber der sie sich als Alternative positionieren will.

Die vorliegende Studie widmet sich folgend einer bestimmten Art von Gegenöffentlichkeit, nämlich alternativen Nachrichtenmedien, die als Gegenpol zur massenmedial hergestellten Öffentlichkeit professioneller, etablierter Nachrichtenmedien betrachtet werden. Als Medien werden in diesem Zusammenhang in Anlehnung an Luhmann (2017 [1995], S. 10) und Saxer (1999, S. 5f.) Kommunikationskanäle betrachtet, die mittels technischer Möglichkeiten Zeichensysteme (im Sinne aktueller Nachrichten) transportieren, in die Gesellschaft eingebunden sind und durch ihren organisationalen Charakter bestimmte Ziele verfolgen. Ein Fokus wird dabei auf alternative Nachrichtenmedien in Zeiten der Digitalisierung (oder des digitalen Strukturwandels) gelegt. Theoretische Beiträge zum digitalen Strukturwandel gehen - wie in Kapitel II.3 erörtert - davon aus, dass durch die Ausdifferenzierung des Mediensystems und die Etablierung digitaler Plattformen (Social Media) mit deren spezifischen («sozialen») Medienlogiken die öffentlichen Kommunikationsmöglichkeiten alternativer Akteur_innen angestiegen sind. Die «Longtail»-Metapher der Öffentlichkeit skizziert ebendies, wonach neben reichweitenstarken Massenmedien bzw. professionellen Informationsanbietern zunehmend Laienkommunikator_innen im «langen Schweif», zwar mit weniger Reichweiten, aber dennoch öffentlichkeitswirksam, ihre Inhalte aufbereiten und verbreiten können (Eisenegger, 2017; Neuberger, 2009). Dies kann gleichbedeutend als eine wesentliche Chance für den Aufschwung alternativer Medien betrachtet werden.

\section{III.4.1 Alternativmedien im digitalen Zeitalter}

Der digitale Medienwandel hat die öffentliche Kommunikation verändert. Somit befördern das Internet und Social-Media-Plattformen das Entstehen neuer Öffentlichkeiten, die in Konkurrenz zu Angeboten massenmedialer Öffentlichkeit stehen können (Neuberger, 2009). Nicht verwunderlich wird in der aktuellen For- 
schung auch ein zunehmendes Interesse an «Alternativmedien» oder «alternativen Nachrichtenmedien» offenbar - wobei letzte Begrifflichkeit im Rahmen der vorliegenden Studie vorgezogen wird, da die aktive und regelmässige Verbreitung von Nachrichten dieser im Vordergrund steht ${ }^{3}$.

Gängige und aktuelle Definitionen des Begriffs «Alternativmedien» betonen vor allem die relationale (vgl. hierzu Holt, 2020; Holt, Figenschou \& Frischlich, 2019) Bedeutung des Begriffes, wonach sich ebendiese Medien als Alternative oder Opposition gegenüber bestimmten Akteur_innen - dem «Mainstream»sehen (Chan, 2017). Alternativmedien stehen demnach in direkter Opposition zu etablierten Informationsmedien und der gesellschaftlichen Elite, nicht nur aus der Medienlandschaft, sondern auch aus Wissenschaft und Politik (Meyers, 2008; Sandoval, 2011; Schweiger, 2017). Der Begriff «Gegenöffentlichkeit» kann in diesem Kontext auch als Kampfbegriff betrachtet werden, der sich gegen die Massenmedien und politische Eliten richtet, bis hin zu einem Vorwurf der Manipulation von Öffentlichkeit dieser medialen und politischen Autoritäten $(\mathrm{Oy}$, 2003; Stamm, 1988, S. 40). Massenmedien würden demzufolge - so Chomsky (2003, S. 123) den gesellschaftlichen Konsens staatlicher und wirtschaftlicher Elite repräsentieren, von dem Randpositionen ausgeschlossen seien (vgl. hierzu auch Scholl, 2009).

Bei alternativen Medien handelt es sich vorwiegend um nichtprofessionelle Medien von Bürger_innen, Protestgruppen, Lai_innen oder politischen Randorganisationen, weshalb sie meist ohne journalistische Qualifikation ausserhalb der Netzwerke von «Mainstreammedien», also etablierten Nachrichtenmedien, produziert werden (Atton, 2011; Chan, 2017; Fuchs, 2010). Dabei sind sie nicht nur durch ihre partizipative Form im Sinne von «Prosumern» (Bruns, 2009), also gleichzeitigen Produzierenden und Konsumierenden, zu charakterisieren, sondern vor allem aufgrund ihrer kritischen Auftrittsweise (Sandoval \& Fuchs, 2010). Die Kritik liegt hier vorwiegend im Infragestellen der dominanten Gesellschaft, was auch mit Protest verbunden sein kann (Fuchs, 2010). Im Fokus steht dementsprechend meist die Imbalance von Machtverteilungen des Mainstreams, also der hegemonialen Öffentlichkeit aus Medien und Politik, weshalb alternative Medien häufig für sozialen und politischen Wandel einstehen und politische Institutionen konfrontieren (Atton, 2011; Haas, 2004). Die Ideologien sind häufig politisch orientiert und richten sich gegen das «Establishment» (Chan, 2017). Ihre Organisationsform ist per Definition in der Regel anti-hierarchisch und nicht kommerziell, wodurch sich auch die «alternative» Form ergibt (Atton, 2011). Ebenso als «alternativ» im Vergleich zum etablierten Journalismus sind die thematisierten

3 Der Begriff «Alternativmedien» wird in dieser Arbeit daher synonym für «alternative Nachrichtenmedien» verwendet, wobei letzterer Begriff dazu dient, das zu analysierende Phänomen genauer zu beschreiben. 
- meist kritischen - Inhalte und genutzten Quellen sowie Kanäle einzustufen (Rauch, 2007, 2016). Dabei nehmen sie per Selbstverständnis eine Stimme oder den Blickwinkel von ignorierten oder unterrepräsentierten Bürger_innen ein (Atton, 2007). Das Publikum alternativer Medien setzt sich folglich gleichbedeutend aus Personen zusammen, die mit der herrschenden Öffentlichkeit, dem Mainstream, unzufrieden sind (Harcup, 2016). Weiter kann die Produktion und Konsumtion von alternativen Nachrichtenmedien ausserdem mit Bürger_innen-Aktivismus in Verbindung stehen (Figenschou \& Ihlebæk, 2018; Haller \& Holt, 2018; Rauch, 2007).

Strittig ist, ob Kriterien wie eine nichtkommerzielle Ausrichtung tatsächlich zutreffen müssen, um als alternatives Nachrichtenmedium zu gelten, oder ob eine Selbstbezeichnung als «alternativ» hierfür genügt (Rauch, 2007). Auch kann eine dichotome Unterscheidung zwischen alternativen und etablierten Nachrichtenmedien kritisch hinterfragt werden, da davon auszugehen ist, dass vor allem in digitalen Öffentlichkeiten hybride Formen alternativer Medien existieren und sich diese in einem breiten Spektrum bewegen (Rauch, 2016). So sieht beispielsweise Downing (2001) ${ }^{4}$ die Verwendung des Begriffes «alternativ» selbst als eine Art Oxymoron, da alles in irgendeiner Form alternativ zu etwas anderem erscheinen könne. In der aktuellen Forschung herrscht Einigkeit darüber, dass alternative und etablierte Nachrichtenmedien innerhalb eines Kontinuums betrachtet werden müssen (vgl. z. B. Holt et al., 2019).

Aktuelle Studien kritisieren zudem eine stark normativ geleitete Herangehensweise an das Phänomen, wonach alternative Nachrichtenmedien vor allem durch ihren progressiven Widerstand gegen den hegemonialen Diskurs definiert würden. Unterschiedliche Erscheinungsformen alternativer Nachrichtenmedien und einschlägige Phänomene in hybriden Mediensystemen würden durch diese theoretische Limitierung nicht miteinbegriffen werden (Holt et al., 2019). Holt, Figenschou und Frischlich gehen in diesem Zusammenhang von einem relationalen, nicht ideologischen Verständnis alternativer Nachrichtenmedien auf der Mikro-, Meso- und Makro-Ebene aus und nehmen mit ihrer Studie eine zentrale Position in der Forschung rund um alternative Nachrichtenmedien ein. Ihrer Definition zufolge zeichnen sich alternative Nachrichtenmedien durch ihre selbst wahrgenommene Korrektivfunktion gegenüber traditionellen Leit- oder «Mainstreammedien» aus. Die Alternative ergibt sich dabei in unterschiedlicher Ausprägung, beispielsweise aufgrund alternativer Kommunikator_innen oder Produzent_innen der Inhalte, die andere Meinungen und Stimmen im Vergleich zum Mainstream vertreten und dadurch alternative Inhalte produzieren (Mikroebene) oder aber aufgrund alternativer Organisationsformen und Publikationsroutinen

4 Hierzu auch in ähnlicher Weise Luhmann (2016 [1996], S. 104): «Das Geheimnis der Alternativen ist: daß sie gar keine Alternative anzubieten haben.» 
von etablierten Netzwerken traditioneller Nachrichtenmedien zu unterscheiden sind (Mesoebene). Auf der Makroebene ergibt sich die Alternative aufgrund der Einnahme als Opposition zu klassischen Nachrichtenmedien auf Ebene des gesamten Mediensystems. Es handelt sich den Autor_innen zufolge um einen relationalen Ansatz, wobei sich die Alternative immer in Opposition zu einer Hegemonie ergebe (Holt et al., 2019).

Obwohl Alternativmedien eine lange historische Tradition aufweisen, werden sie im aktuellen Forschungsstand, der einen Fokus auf moderne, digitale Gesellschaften legt, mit vergleichsweise unterschiedlichen Attributen versehen. Während die Meinungspresse der Französischen Revolution wie auch die Alternativpresse der neuen sozialen Bewegungen der 1960er und 1970er Jahre meist durch eine politische Links-Positionierung, fokussierend auf soziale Gerechtigkeit und sozialen Wandel, gekennzeichnet waren, scheint aktuell das genaue Gegenteil zuzutreffen. Alternative Nachrichtenmedien werden meist mit einer rechtspopulistischen Haltung konnotiert, im amerikanischen wie auch europäischen respektive deutschsprachigen Raum (z. B. Atkinson \& Leon Berg, 2012; Haller, Holt \& de La Brosse, 2019; Holt, 2018, 2020; Padovani, 2016; Schulze, 2020; Zannettou et al., 2017). So hebt beispielweise Holt (2020) zwar hervor, dass alternative Nachrichtenmedien sowohl im politisch linken als auch im politisch rechten Spektrum vorzufinden seien, bezieht sich aber mit Blick auf gesellschaftspolitische Entwicklungen der vergangenen Jahre in seinem Werk vor allem auf rechtspopulistische, alternative Nachrichtenmedien. Der Autor führt dabei beispielhaft die Migrationskritik in unterschiedlichen Ländern an, die mit einer Kritik traditioneller Medien konnotiert gewesen sei. Darunter beispielweise durch die Partei «Alternative für Deutschland» (AfD) und ihrem Kampfbegriff der «Pinocchio Presse», oder die Freiheitliche Partei Österreichs (FPÖ), die mehrmals den öffentlich-rechtlichen Rundfunk (ORF) kritisierte.

Weiter werden in der aktuellen Forschung alternative Medien häufig mit desinformativen Plattformen respektive der Verbreitung desinformativer und verschwörungstheoretischer Inhalte konnotiert, weshalb sie mehrheitlich als Gefahr für die Demokratie betrachtet werden. So kann sich die Kritik gegen das System auch in Form einer Unterstellung gegenüber professionellen Nachrichtenmedien äussern, dass diese «Fake News» verbreiten würden (Holt, 2018); oder aber sie publizieren selbst desinformative Inhalte (Bennett \& Livingstone, 2018; Figenschou \& Ihlebæk, 2018; Hollander, 2018; Zannettou et al., 2017). Die folgenden Subkapitel widmen sich daher detailliert den Themen Desinformation und Verschwörung, da auch diese einen Aufschwung in digitalen Öffentlichkeiten erlangt haben und eine zentrale Rolle einzunehmen scheinen, wenn aktuell über alternative Medien gesprochen wird. 


\section{III.4.2 «Fake News», Desinformation und alternative Nachrichtenmedien}

Der «Fake-News»-Diskurs erwies sich in den vergangenen Jahren als ein relevantes Thema nicht nur in der klassischen Medienberichterstattung, sondern auch in der aktuellen theoretischen und empirischen Forschung. Vor allem Donald Trumps Präsidentschaftswahlkampf gegen Hillary Clinton im Jahr 2016 scheint dabei Anstoss für neues Forschungsinteresse an der absichtlichen Lüge in der öffentlichen Kommunikation gegeben zu haben. Im US-Wahlkampf konnte schliesslich belegt werden, dass insbesondere politische Falschnachrichten - und das vor allem auf Social Media - zirkulierten (Allcott \& Gentzkow, 2017; Bakir \& McStay, 2018). Kritisiert wurde hierbei vor allem das personifizierte, automatisierte Verbreiten von einschlägigen Nachrichten - das Microtargeting - unter Verwendung persönlicher, von Social-Media-Nutzerprofilen abgeleiteter Daten, wie es offenbar auch beim Brexit-Wahlkampf eingesetzt wurde (Bakir \& McStay, 2018). Empirisch nicht belegt werden konnte, ob der gezielte Einsatz von Targeting und Falschnachrichten die Wahlausgänge tatsächlich beeinflusst hat (vgl. hierzu auch Schwaiger, 2019). Historisch betrachtet sind desinformative Nachrichten allerdings kein Phänomen der digitalen öffentlichen Kommunikation, werden sie doch seit jeher mit dem Ziel politischer Einflussnahme oder Propaganda strategisch eingesetzt. Die Wurzeln liegen indes im 19. Jahrhundert, als der Einsatz von «fakes» (oder auch «hoaxes», «humbugs») noch dazu diente, Nachrichten im Sinne einer literarischen Stilfigur lebendiger und ausschmückender zu präsentieren. Dies galt allerdings ab der Jahrhundertwende als verpönt, obgleich hier eine definitorische Grenze zur Satire gezogen werden muss, die in der Regel jedoch nicht die Rezipierenden in die Irre führt, wenn auch mit falschen Informationen gespielt wird, die meist aber durch die Macher_innen selbst transparent gemacht werden. Abgesehen davon galt die Verbreitung von Falschnachrichten spätestens vor Ausbruch des Ersten Weltkrieges als illegitimes Mittel; journalistische Standards mussten eingehalten werden, andernfalls wurde man als «Lügner_in» bezeichnet (Barth \& Homberg, 2018). Dies bedeutet gleichwohl nicht, dass die Verbreitung von Falschnachrichten seither nicht mehr praktiziert wurde. Die bereits erwähnte politische Instrumentalisierung desinformativer Inhalte zeigte sich aus historischer Sicht vor allem in Krisenphasen und Zeiten politischer Umbrüche, in denen Lügen zur Delegitimierung bestimmter Akteur_innen wie auch zur Manipulation der Bevölkerung eingesetzt wurden - und nach wie vor werden (Schiffrin, 2017). Bedeutung oder eine neue Rahmung hat ebendiese Verbreitung von desinformativen Nachrichten im Zuge der digitalen Transformation der Öffentlichkeit gewonnen, einerseits aus den bereits erwähnten Gründen der politischen Instrumentalisierung über digitale Plattformen, z. B. im US-Wahlkampf oder vor der Brexit-Abstimmung, andererseits aufgrund der nicht vorhandenen Barrieren für Lai_innen, Beiträge wie Social Media ohne journalistische Über- 
prüfung zu verbreiten. Nicht verwunderlich ist daher die Sorge, dass alternative Nachrichtenmedien die Verbreitung von Falschnachrichten befördern, da diese sich vor allem in Umbruchsphasen als Opposition zum Establishment betrachten und ebenfalls nicht professionell agieren. Bevor auf den aktuellen Forschungsstand eingegangen wird, bedarf es an dieser Stelle zunächst einiger Begriffsdefinitionen und -abgrenzungen.

Unter «Fake News» können zweierlei Dinge verstanden werden: Erstens die absichtliche Verbreitung von Falschnachrichten, zweitens die Verwendung des Begriffes «Fake News» als Kampfbegriff, um andere Akteur_innen zu delegitimieren. Aber auch jede Bedeutung für sich bringt definitorische Herausforderungen mit sich, und von einheitlichen Definitionen in der aktuellen Forschung kann nicht gesprochen werden. So wurde eine Vielzahl an empirischen Beiträgen zum Thema publiziert, obwohl eine klare Begriffsdefinition kaum erkennbar war' ${ }^{5}$. In einem häufig zitierten Artikel von Allcott und Gentzkow (2017) werden «Fake News» als intentional und nachweisbar falsche Nachrichten definiert, mit dem Ziel, Leser_innen potentiell in die Irre zu führen. Dementsprechend sind sie von irrtümlichen Falschnachrichten abzugrenzen, die unabsichtlich publiziert wurden, wie auch von Gerüchten, Verschwörungstheorien oder Satire (sofern diese nicht missverstanden wird und in die Irre führt). Was genau die Autoren unter «in die Irre führen» verstehen, bleibt unklar und impliziert damit viel Deutungsspielraum. In ähnlicher Weise und dennoch nicht trennscharf genug definieren Bakir und McStay (2018) «Fake News» als gänzlich falsche oder zumindest inhaltlich und/oder kontextuell falsche Nachrichten. Auch Motive hinter der Verbreitung von «Fake News» können Teil von entsprechenden Begriffsdefinitionen sein, wie etwa das Erreichen politischer Ziele (Bennett \& Livingstone, 2018). Oder aber die Darstellungsform der Falschnachricht wird als wesentliches Kriterium gesehen, indem sie professionelle, traditionelle Informationsmedien zu imitieren versucht (Gelfert, 2018; Tandoc, Lim \& Ling, 2017).

Viele dieser Definitionsmerkmale sind einleuchtend, wenngleich ein Spektrum an Interpretationsspielraum bestimmter Kriterien unbestreitbar ist. Dies wird vor allem dann relevant, wenn das Phänomen der Desinformation in empirischen Studien untersucht wird und unterschiedliche Definitionskriterien zu unterschiedlichen Ergebnissen führen können. Eine theoretische Herleitung des Begriffes ist daher unabdingbar. So gibt es Ansätze, Desinformation theoretisch von der «Lüge» abzuleiten (Schwaiger, 2021a; Zimmermann \& Kohring, 2018). Georg Simmels Werke zum «Geheimnis» (1968 [1908]) und der «Psychologie und Soziologie der Lüge» (1992 [1899]) zeigen wesentliche Kriterien der Lüge auf, aber auch davon ausgehende gesamtgesellschaftliche Implikationen, die auf die Lüge im Netz anwendbar sind. Simmel (1968 [1908]) hebt insbesondere die Täuschungs-

5 Der folgende Abschnitt bezieht sich insbesondere auf Schwaiger (2021a). 
absicht hinter der Lüge hervor, wonach es ein Motiv hinter der Verbreitung der Lüge geben müsse und das Geheime oder Lügenhafte bereits dadurch erfüllt sei, dass wesentliche Inhalte verheimlicht würden und somit ein falsches Verständnis der Wirklichkeit bei den Rezipierenden erzeugt werde. Dies impliziert auch eine Intention hinter dem Verbreiten falscher Tatsachen, z. B. um ein Machtgefälle zwischen den Verbreitenden und den Rezipierenden zu erzeugen. Personen mit einem geringeren Wissensschatz sind entsprechend im Nachteil und bestimmte Interessen können durchgesetzt werden (Simmel, 1968 [1908], 1992 [1899]). Essentiell ist Simmel zufolge eine unwahrhaftige, unsittliche Absicht, die im Kontrast zur aufrichtigen Offenbarung der Wahrheit steht. Simmels Ausführungen folgend impliziert die Lüge ein breites Spektrum, das bereits bei dem intentionalen Verbergen wesentlicher Informationen, die für die Einordnung des Sachverhaltes essentiell sind, beginnt. Probleme auf gesamtgesellschaftlicher Ebene ergeben sich laut Simmel (1992 [1899]) vor allem deshalb, weil das Vertrauen als essentieller Faktor für die soziale Ordnung und damit einhergehend die gegenseitige Erwartungssicherheit brüchig wird (vgl. hierzu auch Schwaiger, 2021a). Auch Zimmermann und Kohring (2018) kritisieren Begriffsunschärfen in der Desinformationsforschung und nähern sich theoretisch einer trennscharfen Definition. In ihrem essentiellen Beitrag plädieren sie - berechtigterweise - zunächst für die Verwendung des Begriffes der «aktuellen Desinformation» anstelle von «Fake News», da letzterer Begriff auch als politischer Kampfbegriff, also zur Delegitimierung oppositioneller Akteur_innen, eingesetzt werde. Als wesentliche Definitionsmerkmale erachten die Autoren die Aktualität der Information, im Sinne eines Neuigkeitswertes der Informationen, sowie deren öffentliche Relevanz, den Wahrheitsanspruch der Aussage, die empirische Unwahrheit dieser sowie die Unwahrhaftigkeit, also eine Vorsätzlichkeit hinter der Verbreitung der Desinformation. Eine Täuschungsabsicht wird zwar von den Autoren diskutiert, jedoch als ein zu einschränkendes Definitionsmerkmal erachtet, unter der Annahme, es gebe sowohl täuschende als auch nichttäuschende Desinformationen. Letzterer Punkt ist - vor allem mit Rückbezug auf Simmels Ausführungen - in Frage zu stellen, zumal die Täuschungsabsicht Indizien für die Motive der Verbreitung von Falschinformationen gäbe, die schliesslich substantiell für eine gesamtgesellschaftliche Betrachtung sind. Dennoch ist Zimmermanns und Kohrings (2018) Definition als wesentlich für die aktuelle Forschung zum Thema Desinformation zu erachten.

Von dieser Begriffsdefinition zu unterscheiden ist die Verwendung des Begriffes «Fake News» selbst. Vor allem Rechtspopulist_innen bedienen sich selbst dieses Begriffes, um oppositionelle Parteien und Akteur_innen, so auch etablierte Medien selbst, zu diskreditieren (Barth \& Homberg, 2018; Brumette, DiStaso, Vafeiadis \& Messner, 2018; Zimmermann \& Kohring, 2018). In ähnlicher Weise fungiert auch der Begriff «Lügenpresse» der deutschen Bewegung PEGIDA, um - im Kontext des Migrationsdiskurses - Protest gegenüber den klassischen Me- 
dien zu äussern (Ullrich, 2017). Ebenso gibt es Evidenzen, dass auch alternative Nachrichtenmedien politisch motivierten Gebrauch vom «Fake-News»-Begriff machen, um angebliche Lügen des «Mainstreams» aufzudecken. Dabei handelt es sich bei einschlägigen Beiträgen häufig selbst um Falschinformationen, die zur Diskreditierung eingesetzt werden (Schwaiger, 2021a, 2021b). Auch diese Form des Einsatzes von «Fake News» schürt eine Polarisierung der Gesellschaft, da das Vertrauen in öffentlich agierende Akteur_innen, wie beispielsweise Politiker_innen oder Medienschaffende, und in weiterer Folge eine funktionierende Demokratie ins Wanken gerät. Diesbezüglich ist die Annahme einer als «Post-Truth» bezeichneten Ära (oder eines «postfaktischen Zeitalters») treffend, wonach nicht mehr empirisch wahre Fakten im politischen Diskurs herangezogen werden und die demokratische Basis darstellen, sondern so genannte «alternative Fakten», also nicht auf Wahrheit basierende Informationen, die Aufmerksamkeit in der Öffentlichkeit erregen (Corner, 2017; Hendricks \& Vestergaard, 2018; Lewandowsky, Ecker \& Cook, 2017).

Wie bereits angedeutet, sind es vor allem soziale Medien, die mit dem Aufstieg von desinformativen Beiträgen im öffentlichen Diskurs in Verbindung gebracht werden. Entsprechend gross ist das aktuelle empirische Forschungsinteresse an dieser Thematik. Eine strukturierte Analyse einschlägiger Studien verdeutlicht jedoch, dass viele kontrastierende Studienergebnisse existieren. Dies scheint vor allem darin begründet zu liegen, dass einerseits definitorische Unschärfen hinsichtlich des Phänomens bestehen und andererseits methodische Schwierigkeiten bei der Analyse von digitalen Plattformen existieren (vgl. hierzu IV.2.1).

Zusammenfassend kann von zwei Lagern gesprochen werden, die entweder Gefahren hinsichtlich der Verbreitung von desinformativen Inhalten empirisch belegen und prognostizieren, oder aber von einer geringen Ausbreitung einschlägiger Inhalte ausgehen. «Gefahren» beziehen sich dabei auf die Folgen für die politische Meinungsbildung, die Demokratie und schliesslich die soziale Ordnung als Ganzes. Eine Vielzahl aktueller Studien zum Thema Desinformation beschäftigt sich mit dem US-Wahlkampf bzw. bezieht sich auf Daten, die im Wahlkampf 2016 z. B. auf Twitter oder Facebook entstanden sind. So gehen Allcott et al. (2019) beispielsweise davon aus, dass sowohl auf Twitter als auch auf Facebook die Nutzer_innen-Interaktionen mit desinformativen Inhalten im Zuge des Wahlkampfes gestiegen sind, nach dem Wahlkampf zumindest auf Twitter sogar noch weiter. Die Autoren analysieren konkret die Interaktionen mit einem vordefinierten Sample von 500 als «Fake-News-Sites» bezeichneten Websites. Nicht eindeutig belegt wird dabei, wie ebendiese Seiten definiert werden bzw. welche Kriterien eine Website aufweisen muss, um Teil des Samples zu werden. Es ist zudem anzunehmen, dass viele potentiell desinformative Inhalte auf den Plattformen nicht in die Analyse miteingeflossen sind, da sie nicht von einer dieser Seiten stammen. Törnberg (2018) zeigt weiter in seiner Studie, dass sich desinformative Inhalte vor 
allem in homophilen Gruppen, oder «Echokammern», stärker verbreiten. Eine Art «Entwarnung» geben Studien wie jene von Guess et al. (2019; 2018) mit einem Fokus auf die Plattform Facebook, Grinberg et al. (2019) mit ihrer Studie auf Twitter oder Nelsons und Tanejas (2018) Forschung auf unterschiedlichen mobilen und Desktop-Plattformen. Die Studien kommen allesamt zu der Erkenntnis, dass nur ein geringer Prozentsatz der Nutzer_innen mit desinformativen Inhalten überhaupt in Berührung kommt. Guess et al. (2019) stellen zudem fest, dass, wenn überhaupt desinformative Inhalte geteilt werden, dies meist von Rechtskonservativen und Personen über 65 Jahren geschieht. Auch in dieser Studie wird auf eine vordefinierte Liste von «Fake-News-Domains» als Untersuchungsgrundlage verwiesen, was aus oben genannten Gründen kritisiert werden könnte. So belegen auch Grinberg et al. (2019), dass sich das Publikum von desinformativen Quellen durch ein höheres Alter und rechtskonservative Einstellungen auszeichnet, während Personen mit unterschiedlichen politischen Einstellungen politische Nachrichten hauptsächlich vom «Mainstream» rezipieren. Trotzdem gehen die Autor_innen davon aus, dass nur $1 \%$ der Nutzer_innen mit $80 \%$ der Falschnachrichten konfrontiert werden. Nelson und Taneja (2018) unterscheiden in diesem Zusammenhang weiter zwischen «light internet users» und «heavy users». Während Personen, die weniger Zeit im Internet verbringen, hauptsächlich mit seriösen, etablierten Nachrichten konfrontiert werden, konnten bei starken Internet-Nutzer_innen auch desinformative Inhalte am so genannten «Longtail» der Nachrichtenmedien identifiziert werden. Letztere Gruppe scheint allerdings vergleichsweise klein zu sein. Den eher geringen Stellenwert von Desinformation im Netz bestätigen auch Vargo, Guo und Amazeen (2018), wenngleich die Autor_innen in ihrer Studie aufzeigen, dass zumindest parteiische Medien in den USA die Themenagenden von «Fake-News-Sites» teilweise annehmen, nicht aber die restliche Medienlandschaft. Weiter kommen die Autor_innen zu dem Ergebnis, dass im Vergleich zu desinformativen Inhalten die Verbreitung von «Fact-Checkers», also Beiträgen, die potentielle Falschmeldungen aufdecken, geringer ist.

Dieses Aufdecken von desinformativen Inhalten, das «Debunking», stellt einen weiteren Schwerpunkt der aktuellen Forschung zum Thema «Desinformation» dar. Dabei sind die Ergebnisse aus einer demokratietheoretischen Perspektive ernüchternd und bestätigen meist die eben zitierte Studie von Vargo et al. (2018). So gehen auch Pennycook, Cannon und Rand (2018) davon aus, dass das Debunking auch auf Social Media, beispielweise indem Falschnachrichten sichtlich markiert werden, weniger erfolgsversprechend ist und der primäre Konsum von Falschinformationen dauerhaft die individuellen Einstellungen, z. B. im politischen Bereich, formen kann. Psychologische Studien zeigen weiter, dass sich die ursprünglich konsumierte Nachricht im Gedächtnis verankert und die Aufdeckung der Falschnachricht entsprechend weniger Aufmerksamkeit erhält (Johnson \& Seifert, 1994). Viele Forschende sehen daher aktuell auch die Digital- 
plattformen selbst in stärkerer Verantwortung, um eine potentiell ausgehende Gefahr von Desinformation zu minimieren. Seit einigen Jahren kommunizieren die Plattformen selbst mehr und mehr Bestrebungen, dass Beiträge von Faktencheckern überprüft oder die jeweiligen Algorithmen so adaptiert würden, dass Falschnachrichten weniger prominent in den Newsfeeds der Nutzer_innen erscheinen bzw. nicht mehr algorithmenbasiert weiterempfohlen würden (Schwaiger, 2019). Eine Black-Box stellt nach wie vor die Verbreitung von Nachrichten via Messenger-Diensten dar (z. B. Facebook Messenger, WhatsApp, Telegram), da hier auch der Forschung Grenzen gesetzt werden und nicht nachvollziehbar ist, inwiefern sich desinformative Inhalte in der privaten Kommunikation verbreiten, die in den letzten Jahr immer mehr an Bedeutung gewinnt (Eisenegger, 2019).

\section{III.4.3 Verschwörungstheorien auf alternativen Nachrichtenmedien}

Wie «Fake News» (bzw. die absichtliche Desinformation) insbesondere auf sozialen Medien eine immer grösser werdende Rolle zu spielen scheinen, wird auch das damit verwandte Phänomen der «Verschwörungstheorien» medial, aber auch in wissenschaftlicher Literatur, mit alternativen Medien in Zusammenhang gebracht. Dies liegt vor allem daran, dass auch Verschwörungstheorien der Welt alternative Deutungsmuster offenbaren und somit eine Opposition zur öffentlichen Meinung einnehmen, wovon Alternativmedien mitunter gekennzeichnet sind. Verschwörungstheorien gehen definitorisch davon aus, dass gesellschaftliche Ereignisse miteinander verknüpft sind und von wenigen (meist elitären) Akteur_innen im Geheimen gesteuert werden (Bale, 2007; Castanho Silva, Vegetti \& Littvay, 2017; Clarke, 2002; Douglas, Sutton \& Cichocka, 2017). Insofern sind sie klar von der absichtlichen, aktuellen Desinformation im Sinne von «Fake News» abzugrenzen, da einschlägige Theorien zwar falsch sein können, die Verbreiter innen von Verschwörungstheorien sich aber nicht deren Unwahrheit bewusst sind bzw. noch viel mehr der festen Überzeugung sind, dass erstens jene Theorien die Wahrheit abbilden würden und zweitens sie der Gesellschaft etwas Gutes täten, wenn möglichst viele Personen von deren Richtigkeit überzeugt werden. Aus einer wissenssoziologischen Perspektive, so Anton (2014), ist der Wahrheitsgehalt einer Verschwörungstheorie ausserdem nicht per se festgelegt. Anton (2014, S. 6) unterscheidet in diesem Zusammenhang zwischen «heterodoxen» und «orthodoxen Verschwörungstheorien». Heterodoxe Verschwörungstheorien zeichnet die Annahme von historischen und gesellschaftlichen Entwicklungen oder Ereignissen als Resultat einer Verschwörung aus, die allerdings in den Leitmedien, der breiten Bevölkerung und legitimierenden Instanzen nicht anerkannt ist. Orthodoxe Verschwörungstheorien wiederum werden von ebendiesen Akteur_innen und Deutungsinstanzen als legitimes Erklärungsmodell akzeptiert. So existieren historisch betrachtet (denn wie die intendierte Lüge sind auch Verschwörungstheorien 
aus historischer Sicht kein neuartiges Phänomen, das durch den digitalen Wandel bedingt ist) durchaus ursprüngliche Verschwörungstheorien, die sich als wahr herausstellten, wie beispielsweise die Watergate-Affäre in den 1970er Jahren, obgleich Fälle wie diese meist die Ausnahme darstellen (van Prooijen \& Douglas, 2018). In unserem kulturellen Kreis ist daher der Begriff «Verschwörungstheorie» hauptsächlich mit einer delegitimierenden, abwertenden Zuschreibung versehen, Narrative gelten als irrational und nicht basierend auf empirischen Fakten (Anton et al., 2014; Coady, 2003; Konkes \& Lester, 2015). In modernen Gesellschaften können Verschwörungstheorien als eine Art «Ersatzreligion» gedeutet werden, da in säkularisierten Gesellschaften der Glaube an göttliche Fügungen an Bedeutung verliert (Robertson, 2016). Sie richten sie sich meist gegen Eliten, z. B. aus den Bereichen der Politik, Wirtschaft oder der etablierten Medien. Zugkraft gewinnen sie dann, wenn offizielle Statements von Akteur_innen dieser Felder nicht mehr zufriedenstellend sind, Fragen nicht ausreichend beantwortet werden oder neue Fragen aufgeworfen werden, die in einer Kritik an der Regierung und anderen Akteur_innen münden und den Drang alternativer Erklärungsmodelle forcieren (Konkes \& Lester, 2015). Nicht verwunderlich ist daher, dass Verschwörungstheorien vor allem in Zeiten gesellschaftlicher Krisen breiter in der Öffentlichkeit zirkulieren, was auch mit den Theorien des sozialen Wandels, die in dieser Arbeit bereits besprochen wurden, im Einklang steht (vgl. z. B. Imhof, 1996; Kapitel III.2.1). Gründe hierfür sind Gefühle der Unsicherheit, Angst und Kontrollverlust unter Individuen, die schliesslich nach Antworten für komplexe Sachverhalte suchen (van Prooijen \& Douglas, 2017).

Psychologische Studien kamen weiter zu dem Ergebnis, dass der Glaube an Verschwörungstheorien mit geringem Selbstvertrauen, negativen Einstellungen gegenüber Autoritäten, dem Glauben an Übernatürliches, Paranoia und Schizotypie (Darwin, Neave \& Holmes, 2011) wie auch einem subjektiven Gefühl der Unsicherheit und Misstrauen gegenüber der Regierung korreliert (Bode \& Vraga, 2018). Auch bestimmte politische Einstellungen können die Empfänglichkeit von Verschwörungstheorien positiv beeinflussen. So gibt es in der aktuellen Forschung Evidenzen, dass Populismus, definiert durch eine antizipierte Bürger_innenbezogenheit oder «Volksnähe» und Kritik gegenüber Eliten, mit dem Glauben an Elitenverschwörungen positiv zusammenhängt (Castanho Silva et al., 2017). Dies bestätigen Studien aus den USA, in denen die Nutzung politisch rechtsorientierter Medien (wie in dieser Studie Fox News) und eine damit einhergehende bestimmte politische Einstellung mit dem Glauben an (politisch konforme) Verschwörungstheorien belegt wurde (Hollander, 2018), wie auch eine rechtsautoritaristische Einstellung mit der Unterstützung an Verschwörungstheorien korreliert (Wood \& Gray, 2019). Je nach politischer Einstellung können Verschwörungstheorien von den Rezipient_innen auch in unterschiedlicher Weise - passend zur subjektiven Einstellung - gedeutet werden (Nefes, 2015). Allerdings existieren auch Studien, 
die ebendiese Zusammenhänge nicht belegen konnten, wonach der Einfluss politischer (konservativer) Einstellungen nicht bestätigt, aber die subjektive Affinität gegenüber bisher Ungesehenem bzw. Übernatürlichem als Prädiktor identifiziert wurde (Oliver \& Wood, 2014). Weiter gibt es Evidenzen, dass vor allem Bürger_innen, die glauben, ihnen würden Informationen vorenthalten, eine höhere Affinität hinsichtlich des Glaubens an Verschwörungstheorien besitzen (Gualda \& Rúas, 2019).

In den vergangenen Jahren verlagerte sich die kommunikationswissenschaftliche Forschung zum Phänomen «Verschwörungstheorien» vor allem auf die Fragestellung, inwiefern sich ebendiese auf Social-Media-Plattformen verbreiten. Wie auch im Forschungsstand bezüglich der absichtlichen Desinformation angesprochen, sind einige Ergebnisse jedoch mit Vorsicht zu betrachten, da häufig keine trennscharfe Unterscheidung zwischen Verschwörung, Desinformation oder Gerüchten vorgenommen wird (z. B. Anagnostopoulus et al., 2014; vgl. hierzu auch Hollander, 2018). Prinzipiell hängt die Verbreitung von Verschwörungstheorien auch damit zusammen, dass diese wesentliche Nachrichtenwerte bedienen: Sie sind häufig emotional aufgeladen, skandalisierend oder in anderer Weise affektiv (Konkes \& Lester, 2015) und erzielen dadurch mehr Aufmerksamkeit respektive «User-Engagement» - vor allem im Vergleich zu seriösen, wissenschaftlichen Nachrichten (Zollo et al., 2015). Social-Media-Plattformen können daher als idealer Nährboden für Verschwörungstheorien betrachtet werden, da Algorithmen auf Social Media emotionale Beiträge mit vielen Nutzer_innenreaktionen noch weiter streuen (Schwaiger, 2019, S. 150; Stieglitz \& Dang-Xuan, 2013; Wahl-Jorgensen, 2018). Aus einer netzwerkanalytischen Perspektive scheint zudem die Zugehörigkeit zu einer bestimmten Community bzw. Gruppe von Gleichgesinnten einen wesentlichen Einfluss auf das Engagement hinsichtlich Verschwörungstheorien zu haben. Dieses korreliert mit den Nutzungsmustern von Personen derselben Community und Personen mit ähnlichen Überzeugungen und Einstellungen (im Sinne von polarisierten Communities), wodurch sich vor allem in diesen Kreisen Verschwörungstheorien rasch verbreiten können (Anagnostopoulus et al., 2014; A. Bessi et al., 2015; Alessandro Bessi et al., 2016). So gehen auch Warner und Neville-Shepard (2014) davon aus, dass sogenannte «Echokammern» (Flaxman et al., 2016), also Cluster von Gleichgesinnten, die sich gegenseitig in ihren Meinungen bestärken und kaum oder gar keinen Zugang zu anderen Meinungen mehr haben, den Glauben an Verschwörungstheorien, die in diesen Echokammern zirkulieren, verstärken. An dieser Stelle muss aber darauf hingewiesen werden, dass die aktuelle Forschung hinsichtlich des Echokammern-Phänomens strittig ist und mittlerweile die von Echokammern ausgehenden gesellschaftlichen Gefahren als eher gering einschätzt werden (Dubois \& Blank, 2018; Zuiderveen Borgesius et al., 2016; vgl. Kapitel II.3.3). 
Verschwörungstheorien tragen - selbst wenn allenfalls nur kleine Bevölkerungsgruppen daran glauben - auch gesamtgesellschaftliche Konsequenzen. Empirische Experimentalstudien zeigen beispielsweise, dass bestimmte Verschwörungstheorien das Handeln ihrer Anhänger_innen beeinflussen können. Verschwörungstheorien mit einem Themenfokus auf den Klimawandel könnten demnach dazu führen, dass die Bereitschaft, auf den persönlichen ökologischen Fussabdruck zu achten oder an Petitionen gegen die Klimaerwärmung teilzunehmen, geringer wird (Jolley \& Douglas, 2014; van der Linden, 2015). In ähnlicher Weise hat die Rezeption von regierungskritischen Verschwörungstheorien eine geringere Bereitschaft, sich in der Politik zu engagieren, zur Folge - beides vor allem dann, wenn den Rezipierenden eine aufklärende Information nicht zugänglich ist (Jolley \& Douglas, 2014). Entsprechend relevant ist auch die Frage nach dem «Debunking» von Verschwörungstheorien, also dem Aufdecken falscher Informationen, wofür sich viele (empirische) Studien interessieren. Die diesbezüglich vorliegenden Ergebnisse sind strittig; so gibt es die Annahme, dass ein jeder Versuch, Verschwörungstheorien aufzudecken, für die Anhänger_innen der Theorien ein weiterer Beweis für deren Existenz sei (Kovic, Caspar \& Rauchfleisch, 2019). Es ist davon auszugehen, dass dies vor allem für jene Personen der Fall ist, bei denen sich das Verschwörungsdenken schon fest verankert hat. Ferner kann vermutet werden, dass die Ergebnisse hinsichtlich des Debunkings von absichtlichen Falschnachrichten auch auf das Phänomen «Verschwörungstheorien» zutreffen (vgl. Kapitel. III.4.2).

Zusammenfassend lässt sich festhalten, dass Verschwörungstheorien rein inhaltlich ein wesentliches Thema von alternativen Nachrichtenmedien darstellen können. Sie richten sich - wie es Grunddefinitionen alternativer Nachrichtenmedien implizieren - meist gegen «die Elite», sehen sich demnach als Opposition zum «Mainstream», der öffentlichen Meinung. Nicht verwunderlich ist daher, dass die aktuelle Forschung rund um alternative Nachrichtenmedien Verschwörungstheorien als ein potentielles Element dieser sehen - ähnlich wie die absichtliche Desinformation.

\section{III.4.4 Alles «Fake News» und Verschwörung?}

Wie im vorigen Kapitel deutlich wurde, ist der aktuelle Forschungsstand hinsichtlich alternativer Nachrichtenmedien nicht nur im US-amerikanischen Raum, sondern auch im europäischen und schliesslich deutschsprachigen Raum von einer Konnotation mit erstens rechtspopulistischen Medien und zweitens desinformativen Medien geprägt (z. B. Bennett \& Livingstone, 2018; Figenschou \& Ihlebæk, 2018; Hollander, 2018; Holt, 2020; Zannettou et al., 2017). Die Grenzen scheinen dabei häufig fliessend; so werden oftmals unterschiedliche Phänomene, wie die absichtliche Desinformation oder die Verbreitung von Verschwörungstheorien, 
miteinander vermischt. Die vorliegende Arbeit versucht hiervon aus unterschiedlichen Gründen Abstand zu nehmen. Erstens zeigt die historische Herleitung des Phänomens, dass alternative Medien Ausdruck unterschiedlichster Bewegungen sein können, die entsprechend um verschiedene Themendiskurse strukturiert sind. Wesentliche Beispiele hierfür sind die Meinungspresse im Rahmen der Französischen Revolution oder die Alternativpresse der neuen sozialen Bewegungen. Die ausschliessliche Verwendung des Alternativmedien-Begriffs für rechtspopulistische, desinformative Medien würde daher zu kurz greifen. Nicht nur in der Geschichte konnten politisch linksorientierte Medien verzeichnet werden, sondern auch aktuell - unter der Annahme eines pluralen Mediensystems - ist davon auszugehen, dass eine Vielfalt alternativer Nachrichtenmedien existiert. Zweitens müssen Phänomene wie Rechtspopulismus und Desinformation voneinander abgegrenzt werden. Es kann nicht ungeprüft davon ausgegangen werden, dass ein jedes politisch rechtsorientiertes Nachrichtenmedium Falschmeldungen verbreitet und umgekehrt. Gleichzeitig wird in der Literatur auch der Begriff «Fake-News-Medien» verwendet (z. B. Allcott et al., 2019; Vargo et al., 2018). Dabei sind mehrere Aspekte zu unterscheiden: Einerseits stellt sich die Frage, ob von der Verwendung des «Fake-News»-Begriffes als Kampfbegriff durch die Medien selbst gesprochen wird, oder ob andererseits die Annahme besteht, dass diese Nachrichtenmedien desinformative Inhalte verbreiten. Ausserdem stellt sich die Frage, ab wann ein Medium überhaupt als «Fake-News»-Medium bezeichnet werden kann, erscheint es doch eher unwahrscheinlich, dass Nachrichtenmedien ausschliesslich Falschnachrichten verbreiten. Auch der Begriff der Desinformation selbst muss differenziert betrachtet werden. Wie im vorigen Kapitel definiert, impliziert Desinformation vor allem die absichtliche Verbreitung empirisch falscher, aktueller Nachrichten (Zimmermann \& Kohring, 2018). Verschwörungstheorien können zwar ebenso empirisch falsch sein, die Absicht der Verbreitung einer Falschnachricht muss allerdings nicht unbedingt gegeben sein. So ist es auch potentiell möglich, dass Verbreiter_innen von Verschwörungstheorien zumindest selbst davon überzeugt sind, dass einschlägige Theorien die Wahrheit abbilden und die Menschheit darüber aufklären wollen. Auch greift es zu kurz, Verschwörungstheorien per se im rechten politischen Spektrum anzusiedeln - selbst wenn einschlägige Verschwörungstheorien existieren, die vor allem in rechten Milieus Anklang finden.

Vor allem das Thema «Desinformation» brachte eine eindeutig negative Konnotation hinsichtlich alternativer Medien. Diese werden - im negativen Sinne - als Gegenpol zum professionellen Journalismus betrachtet, der sich an journalistische Standards hält. Der ursprüngliche Gedanke einer Kritik- und Kontrollfunktion gegenüber politischen und auch medialen Eliten wäre allerdings durchaus legitim und noch viel mehr ein wesentlicher Aspekt demokratischer Gesellschaften. Dabei ist eine dichotome Unterscheidung zwischen «guten Main- 
streammedien» und «bösen Alternativmedien» (genauso wie eine Unterscheidung der «klugen Elite» und der «dummen Masse») nicht zuletzt aufgrund der Annahme eines pluralen Mediensystems, das disperse Nachrichtenformate impliziert, nicht haltbar. So sind durchaus auch Online-Newsmedien denkbar, die zwar nicht (oder ggf. noch nicht) zu dem Kreis etablierter Nachrichtenmedien zählen, aber durchaus demokratische Absichten im Sinne partizipativer, informativer Berichterstattung verfolgen. Aus diesem Grund möchte sich die vorliegende Arbeit dem Begriff alternativer Nachrichtenmedien öffnen und explorativ erforschen, in welchen Ausprägungen diese im deutschen Sprachraum überhaupt vorliegen. Erst dann kann eine möglichst trennscharfe Begriffsdefinition vorgenommen werden, die dem digitalen Strukturwandel der Öffentlichkeit gerecht wird. Ebenso ist in diesem Zusammenhang eine Analyse von hoher Relevanz, inwiefern «Alternativ» und «Mainstream» in der Öffentlichkeit selbst, also gleichbedeutend auch für das Publikum, in einer «dichotomen Form» auftreten, also getrennt voneinander agieren und als Teilöffentlichkeiten erscheinen. Aus diesem Grund wird im Rahmen dieses Forschungsprojektes angestrebt, die Relationen zwischen diesen vermeintlichen Gegenpolen in Erfahrung zu bringen. Das folgende Hauptkapitel versucht folglich, eine theoretische Einordnung der Relationen zwischen Öffentlichkeit und Gegenöffentlichkeit mit einem Blick auf Erkenntnisse der relationalen Soziologie vorzunehmen. 